\title{
Early Decompressive Surgery in Traumatic Thoraco-Lumbar Spine: When and Why?
}

Alessandro Landi*, Angela Ambrosone and Roberto Delfini

Department of Neurology and Psychiatry, Division of Neurosurgery, Sapienza University of Rome, Rome, Italy

Keywords: Thoraco-lumbar spine; Timing; Early decompression; Spine trauma

\section{Introduction}

Spinal trauma is a very common disease, associated with spinal cord injury in $15-30 \%$ of cases [1]. The treatment is affected both from non-modifiable variables (fracture's morphology and biomechanics of the trauma, fracture site, neurological status, primary or secondary comorbidities) both from modifiable variables (first aid and hospital transportation, supportive therapy, surgical timing etc.). The role of the surgical timing after acute thoraco-lumbar spinal cord injury is still one of the most controversial points actually debated in literature. Surgical treatment is conditioned both by the general conditions of the patient both by the extent of the neurological deficit. In literature are described three possible windows for surgical timing: early surgery , performed in the first 48 hours; intermediate surgery, performed between 48 hours - 7 days; late surgery, performed after 7 days from the injury. In the light of the debatment actually under discussion in literature, the real question is: The implementations of the early surgery have effectively a role in the management of thoraco-lumbar spine injury and, if so, when is mandatory? Actually, based on the literature evidence, is extremely difficult to find a clear indication.

\section{Thoraco-Lumbar Spinal Cord Injury: Current Concept}

Spinal cord injury (SCI) is one of the primary causes of neurological damage. Actually there isn't a clear opinion about what is the correct timing of surgical decompression in cases of thoraco-lumbar fractures. Surgery is crucial, in order to reduce secondary damage and to improve patient's outcome [2]. Most of the concerns about the timing of treatment are related to insufficient information about the pathophysiology of the damage and the effects of surgical decompression. In fact spinal cord trauma induces a combination of signs and symptoms associated with earlier and later spinal cord damage [1,3-6]. In particular, traction and compression forces onto the spinal cord, can cause a primary damage to the central gray substance according to high metabolic challenges. These damages are irreversible since the first hours from trauma, while the white matter damage becomes irreversible 72 hours after trauma [1].

Experimental studies show that the length of spinal cord compression causes the severity of the pathological changes and the degree of neurological recovery: the longer is length, the smaller is the possibility of clinical recovery [3,7-9]. Surgical decompression potentially allows a reduction of the intradural pressure, an increase in blood flow to the spinal cord, thus reducing the risk of ischemic lesions and preventing secondary damage $[6,10,11]$. However it is unclear what should be the better timing for decompressive surgery.

In literature, there are many works concerning the validity and effectiveness of the implementation of an early surgical treatment (within 8 hours of injury). From a literature review we can assure that an early surgery may be associated with an increasing in intra-operative blood loss and the onset of hypotension, with an increased risk of spinal cord ischemic lesions and then neurological deficit. However it guarantees a better outcome, related to an early mobilization of the patient, a shorter hospitalization and a lower risk of pulmonary complications and thromboembolism $[6,8,11]$. It is important to underline that a non-modifiable factor that influences surgical timing in the presence of systemic complications, such major bleeding, shock, sepsis etc.

The presence of this comorbidities cause an unmodifiable delayed in surgical treatment, which is unavoidable. Some studies have shown that early surgery ( 8 hours of injury) has guaranteed a sudden improvement in neurological status and a better outcome in the subsequent follow-up [7,10-15]. Chenzing et al. [16] have shown that when early surgery is performed, the neurological outcome is closely tied to the neurological pre-operative status. More in details he shows that there is a greater chance of recovery with a stadium ASIA C and D. In their retrospective study Boakye et al. [17] shows that independent variables as age, comorbidities and pre-existing AID score, also influenced the outcome.

Surgical time was the strongest predictor of the outcome. In an early surgery there are a lower percentage of postoperative complications ( $18.7 \%$ vs $25.9 \%$ for the late surgery) and a reduction in hospitalization (7-10 days vs. 12-15 days). La Rosa et al. [6] conducted a systematic review about this topic, and concluded that a decompressive surgery in less than 24 hours from injury can result in a better neurological outcome. On the contrary, some studies show that in cases of complete or incomplete neurological deficit, the neurological improvement is totally independent to the surgical timing. In a prospective study of 106 patients of Pointillart et al. [18], approximately half of the cohort underwent early surgery $(<8$ hours) with no improvement in neurological status. McKinley et al. [19] concluded that there weren't differences in neurological status between early surgery $(<72$ hours from the trauma) and late surgery.

\section{Thoraco-Lumbar Spinal Cord Injury: Our Experience}

In the light of the nebulous horizon in literature concerning this crucial topic, we have tried to obtain concrete data about early surgical decompression in thoraco-lumbar spinal cord injury, with the aim to suggest a better definition of optimal surgical timing in this pathological disease. For this reason, we have conducted a descriptive longitudinal retrospective study on 166 patients with thoracolumbar fractures and neurological deficits. All patients enrolled for our study, underwent surgery between 2002 and 2010 at the Neurosurgery Dpt of University Hospital of Rome "Sapienza". All patients were followed up periodically for a period ranging between three years and the eleven years. We analyzed the following variables: age, sex, AO spine classification of the fracture, type of surgical treatment (posterior approach with minimally invasive or open decompression and stabilization when

*Corresponding author: Alessandro Landi MD, PhD, Department of Neurology and Psychiatry, Division of Neurosurgery, Sapienza University of Rome, Italy, Viale del Policlinico 155, Rome 00181, Tel: +39 06 49979105; E-mail: dott.alessandro.landi@gmail.com

Received April 05, 2016; Accepted April 26, 2016; Published April 28, 2016

Citation: Landi A, Ambrosone A, Delfini R (2016) Early Decompressive Surgery in Traumatic Thoraco-Lumbar Spine: When and Why? J Spine 5: 301. doi:10.4172/2165-7939.1000301

Copyright: $\odot 2016$ Landi A, et al. This is an open-access article distributed under the terms of the Creative Commons Attribution License, which permits unrestricted use, distribution, and reproduction in any medium, provided the original author and source are credited. 
Citation: Landi A, Ambrosone A, Delfini R (2016) Early Decompressive Surgery in Traumatic Thoraco-Lumbar Spine: When and Why? J Spine 5: 301. doi:10.4172/2165-7939.1000301

Page 2 of 3

\begin{tabular}{|c|c|c|c|c|c|c|}
\hline \multirow{3}{*}{ TIMING } & \multirow{3}{*}{ SITE } & AIS SCORE & \multirow{3}{*}{ AO SPINE } & SEX & \multirow{3}{*}{ MEDIUM AGE+DS } & \multirow{3}{*}{ RECOVERY } \\
\hline & & \multirow{2}{*}{ PRE-OP } & & $\mathrm{F}=45(27,5 \%)$ & & \\
\hline & & & & $M=121(72,5 \%)$ & & \\
\hline EARLY 1-2 DAYS & $\mathrm{D}=9(21,4 \%)$ & $E=17(60,5 \%)$ & $A=35(83,3 \%)$ & $\mathrm{F}=12(28,6 \%)$ & 42.3AA & MEDIUM HOSPIT $=23$ \\
\hline \multirow[t]{4}{*}{42 PTS(25,3\%) } & $\mathrm{L}=26(61,9 \%)$ & $\mathrm{D}=7(16,7 \%)$ & $\mathrm{B}=7(16,7 \%)$ & $M=30(71,4 \%)$ & $20,1 \pm D S$ & MEDIUM ICU=17 \\
\hline & $\mathrm{D}-\mathrm{L}=7(16,7 \%)$ & $\mathrm{C}=0$ & $\mathrm{C}=0$ & & & COMPL. $=11 \%$ \\
\hline & & $B=9(21,4 \%)$ & & & & \\
\hline & & $A=9(21,4 \%)$ & & & & \\
\hline INTERMEDIATE & $\mathrm{D}=11(23,4 \%)$ & $E=32(68,1 \%)$ & $A=44(93,6 \%)$ & $\mathrm{F}=8(17 \%)$ & 44,9 & MEDIUM HOSPIT. $=29$ \\
\hline 3-7 DAYS & $\mathrm{L}=24(51,1 \%)$ & $\mathrm{D}=5(10,6 \%)$ & $B=3(6,4 \%)$ & $\mathrm{M}=39(83 \%)$ & $\pm 16,1 \mathrm{DS}$ & MEDIUM ICU=18 \\
\hline \multirow[t]{3}{*}{47 PTS $(28,3 \%)$} & $D-L=12(25 \%)$ & $\mathrm{C}=3(6,4 \%)$ & $\mathrm{C}=0$ & & & COMPL. $=21 \%$ \\
\hline & & $\mathrm{B}=4(8,5 \%)$ & & & & \\
\hline & & $A=3(6,4 \%)$ & & & & \\
\hline LATE & $D=26(33,8 \%)$ & $E=53(68,8 \%)$ & $A=70(90,9 \%)$ & $\mathrm{F}=25(32 \%)$ & 47,6 & MEDIUM HOSPIT: $=37$ \\
\hline >7 DAYS & $\mathrm{L}=34(44,1 \%)$ & $D=14(18,2 \%)$ & $\mathrm{B}=6(7,8 \%)$ & $M=52(67,5 \%)$ & $\pm 16,9 \mathrm{DS}$ & MEDIUM ICU=26 \\
\hline \multirow[t]{3}{*}{77 PTS } & $D-L=17(22,1 \%)$ & $C=4(5,2 \%)$ & $\mathrm{C}=1(1,3 \%)$ & & & COMPL. $=33 \%$ \\
\hline & & $B=3(3,9 \%)$ & & & & \\
\hline & & $A=3(3,9 \%)$ & & & & \\
\hline
\end{tabular}

Table 1: First multivariate analysis in which time surgery was correlated with ASIA, skills and patient outcome, expressed as days of hospitalization and comorbidity.

\begin{tabular}{|c|c|c|c|c|}
\hline \multirow{2}{*}{ AIS SCALE } & \multirow{2}{*}{ SITE } & TIMING:EARLY & INTERMEDIATE & LATE \\
\hline & & 1-2 DAYS & 2-7 DAYS & $>7$ DAYS \\
\hline \multirow[t]{3}{*}{$E=102 P T S$} & $D=32(11,4 \%)$ & 17 PTS & 32PTS & 53 PTS UNCHANGED \\
\hline & $\mathrm{L}=50(80 \%)$ & UNCHANGED =100\% & UNCHANGED $=100 \%$ & $100 \%$ \\
\hline & $\mathrm{DL}=20(19,6 \%)$ & & & \\
\hline \multirow[t]{3}{*}{$\mathrm{D}=26 \mathrm{PTS}$} & $D=5(19,2 \%)$ & 7PT & 5PTS & 14PTS \\
\hline & $\mathrm{L}=15(57,7 \%)$ & UNCHANGED =0 & UNCHANGED=1(20\%) & UNCHANGED=3(21,4\%) \\
\hline & $\mathrm{DL}=6(23,1)$ & IMPR.1ASIA=7(100\%) & IMPR1 ASIA=4(80\%) & IMPR.1ASIA=11(78,6\%) \\
\hline \multirow[t]{4}{*}{$\mathrm{C}=7 \mathrm{PTS}$} & $D=2(28,6 \%)$ & 0 PTS & 3 PTS & 4PTS \\
\hline & $L=4(57,1 \%)$ & & UNCHANGED $1(33,3 \%)$ & UNCHANGED=2(50\%) \\
\hline & $\mathrm{DL}=1(14,3 \%)$ & & IMPR.1ASIA=1(33,3\%) & IMPR.1 ASIA=1(25\%) \\
\hline & & & IMPR.2 ASIA=1(33,3\%) & IMPR.2 ASIA=1(2\%) \\
\hline \multirow[t]{4}{*}{$\mathrm{B}=16 \mathrm{PTS}$} & $\mathrm{D}=4(25 \%)$ & 9PTS & 4PT & 3 PTS \\
\hline & $L=6(37,5 \%)$ & UNCHANGED=1(11,10\%) & UNCHANGED=1(25\%) & UNCHANGED=2(66,7\%), \\
\hline & $\mathrm{DL}=6(37,5 \%)$ & IMPR.1ASIA=3(33,3\%) & IMPR. 1 ASIA=2(50\%) & IMPR.1 ASIA=1(33,3\%) \\
\hline & & IMPR 2ASIA=5(55,6\%) & IMPR. 2 ASIA=1(25\%) & IMPR. 2 ASIA=0 \\
\hline \multirow[t]{3}{*}{$\mathrm{A}=15 \mathrm{PT}$} & $D=3(20 \%)$ & UNCHANGED=100\% & UNCHANGED 100\% & PTS UNCHANGED $=100 \%$ \\
\hline & $L=9(60 \%)$ & & & \\
\hline & $\mathrm{DL}=3(20 \%)$ & & & \\
\hline
\end{tabular}

Table 2: Second multivariate analysis : the change in the neurological status, expressed through AIS score, was related with the surgical timing

performed), surgical timing ( $<48$ hours (early), between 48 and 7 days (intermediate) and $>7$ days (late) and pre and post-operative neurological status, expressed as ASIA-AIS score [20,21]. Cervical trauma wasn't considered in our paper. Osteoporotic and pathological fractures and conservative case were excluded. We conducted three multivariate analyses. These analysis show that, concerning location and type of fracture, early surgical treatment results in a reduction of the median hospital length of stay and ICU stay, as well as in a reduction of cases of nosocomial complications (Table 1). The early surgical treatment appears to provide a better neurological recovery. Better is the ASIA-AIS score on admission, better is the outcome. According to our research, when there are thoracic and lumbar spinal fracture with incomplete neurological deficit, early surgery influences the outcome of patients in terms of better neurological recovery, reduction in hospitalization and reduction of associated comorbidities. The thoracic fractures have the worse outcome. Concerning thoracic location, early treatment seems to have better results: better is the ASIA-AIS score on admission, better is the outcome. Timing doesn't affect the outcome when the ASIA-AIS score is A or E (Table 2). We must implement our research with inclusion of many other variables in relation to the thoracolumbar trauma, which, however, should not be considered in this multivariate analysis. However, the results seems to be encouraged; in fact, according to the scientific literature, seems to suggest that timing of surgery is the most important factor that could influence the patient outcome, in terms of ASIA-AIS score improvement, when there is a preoperative incomplete neurological damage. In case of complete neurological damage or total absence of damages, timing of surgery doesn't affect the outcome.

\section{Conclusion}

Early decompressive surgery in the thoracic and lumbar spine with incomplete neurological damage could positively affect the outcome in terms of neurological recovery, functional restoration, and length of stay and associated comorbidity. Thoracic location of the fracture still has a worst outcome if compared to the other localization, probably because of the vascularization of this segment. Moreover, independently from the localization of the fracture, early surgery seems to have better results when the initial ASIA-AIS score is good; the better the ASIA-AIS score on admission, the better the outcome. Timing doesn't affect the outcome when the ASIA-AIS score is A or E. 
Citation: Landi A, Ambrosone A, Delfini R (2016) Early Decompressive Surgery in Traumatic Thoraco-Lumbar Spine: When and Why? J Spine 5: 301. doi:10.4172/2165-7939.1000301

\section{References}

1. Xing D, Chen Y, Ma JX, Song DH, Wang J, et al. (2013) A methodological systematic review of early versus late stabilization of thoracolumbar spine fractures Eur Spine J 22: 2157-2166.

2. Tator $\mathrm{CH}$ (2006) Review of treatment trials in human spinal cord injury: issues, difficulties, and recommendations. Neurosurgery 59: 957-982.

3. Bracken MB, Holford TR (1993) Effects of timing of methylprednisolone or naloxone administration on recovery ofsegmental and long-tract neurological function in NASCIS 2. J Neurosurgery 79: 500-507.

4. Holmes JF, Miller PQ, Panacek EA, Lin S, Horne NS, et al. (2001) Epidemiology of thoracolumbar spine injury in blunt trauma. Acad Emerg Med 8: 866-872.

5. Launay O, Charles YP, Steib JP (2012) Should post-traumatic thoracolumbar Frankel A paraplegia be operated as an emergency? Report of three cases and review of the literature. Orthop Traumatol Surg Res 98: 352-358.

6. La Rosa G, Conti A, Cardali S, Cacciola F, Tomasello F (2004) Does early decompression improve neurological outcome of spinal cord injured patients? Appraisal of the literature using a meta-analytical approach. Spinal Cord 42 : 503-512.

7. Harrop JS, Sharan A, Ratliff J (2006) Central cord injury: pathophysiology, management, and outcomes. Spine J 6: 198S-206S

8. McLain RF, Sparling E, Benson DR (1993) Early failure of short-segment pedicle instrumentation for thoracolumbar fractures. A preliminary report. J Bone Joint Surg Am 75: 162-167.

9. Schinkel C, Frangen TM, Kmetic A, Andress HJ, Muhr G; German Trauma Registry (2006) Timing of thoracic spine stabilization in trauma patients: impact on clinical course and outcome. J Trauma 61: 156-160.

10. Krengel WF 3rd, Anderson PA, Henley MB (1993) Early stabilization and decompression for incomplete paraplegia due to a thoracic-level spinal cord injury. Spine (Phila Pa 1976) 18: 2080-2087.

11. Maynard FM Jr, Bracken MB, Creasey G, Ditunno JF Jr, Donovan WH, et al (1997) International Standards for Neurological and Functional Classification of Spinal Cord Injury. American Spinal Injury Association. Spinal Cord 35: 266274.
2. Bliemel C, Lefering R, Buecking B, Frink M, Struewer J, et al. (2014) Early or delayed stabilization in severely injured patients with spinal fractures? Current surgical objectivity according to the Trauma Registry of DGU: Treatment of spine injuries in polytrauma patients. J Trauma Acute Care Surg 76: 366-373.

13. Decq $P$, Kéravel $Y$ (1995) Neurochirurgie : traumatismes vertébromédullaires. Prise en charge des 48 premières heures Paris: Universités francophones ed. Paris $370-393$

14. Nyström B, Berglund JE (1988) Spinal cord restitution following compression injuries in rats. Acta Neurol Scand 78: 467-472.

15. Cengiz SL, Kalkan E, Bayir A, llik K, Basefer A (2008) Timing of thoracolomber spine stabilization in trauma patients; impact on neurological outcome and clinical course. A real prospective (rct) randomized controlled study. Arch Orthop Trauma Surg 128: 959-966.

16. Cengiz SL, Kalkan E, Bayir A, Ilik K, Basefer A (2008) Timing of thoracolomba spine stabilization in trauma patients; impact on neurological outcome and clinical course. A real prospective (RCT) randomized controlled study. Arch Orthop Trauma Surg 128: 959-966

17. Boakye M, Arrigo RT, Hayden Gephart MG, Zygourakis CC, Lad S (2012) Retrospective, propensity score-matched cohort study examining timing of fracture fixation for traumatic thoracolumbar fractures. J Neurotrauma 29 2220-2225.

18. Pointillart V, Petitjean ME, Wiart L, Vital JM, Lassié P, et al. (2000) Pharmacological therapy of spinal cord injury during the acute phase. Spinal Cord 38: 71-76.

19. McKinley W, Meade MA, Kirshblum S, Barnard B (2004) Outcomes of early surgical management versus late or no surgical intervention after acute spinal cord injury. Arch Phys Med Rehabil 85: 1818-1825.

20. Rath SA, Kahamba JF, Kretschmer T, Neff U, Richter HP, et al. (2005) Neurological recovery and its influencing factors in thoracic and lumbar spine fractures after surgical decompression and stabilization. Neurosurg Rev 28 : 44-52.

21. Kirshblum SC, Burns SP, Biering-Sorensen F, Donovan W, Graves DE, et al. (2011) International standards for neurological classification of spinal cord injury. J Spinal Cord Med 34: 535-546. 